\title{
EVALUATION OF MYCOBACTERIUM TUBERCULOSIS DRUG SUSCEPTIBILITY TESTING BY DIRECT NITRATE REDUCTASE ASSAY
}

\author{
Neelu Jain'1, Vaishali Jain², Nitin Jain², Saurabh Jain ${ }^{4}$ \\ ${ }^{1}$ Assistant Professor, Department of Microbiology, Shyam Shah Medical College, Rewa. \\ ${ }^{2}$ Consultant, Department of Obstetrics and Gynaecology, Mahaveer Hospital, Indore. \\ ${ }^{3}$ Consultant, Department of Medicine, Bhagyoday Hospital, Sagar. \\ ${ }^{4}$ Consultant, Department of Orthopaedics, Mahaveer Hospital, Indore.
}

\begin{tabular}{l}
\hline ABSTRACT \\
BACKGROUND \\
Drug resistance, multidrug resistance (MDR) and extensive drug resistance (XDR) in tuberculosis is a matter of great concern for \\
TB control programs. There is concern and need for early diagnosis of these multidrug resistant strains for better treatment.
\end{tabular}

AIM

To evaluate Mycobacterium tuberculosis drug susceptibility by Direct Nitrate Reductase assay.

\section{MATERIAL AND METHOD}

We evaluated the usefulness of Nitrate Reductase Assay (NRA) as a tool for rapid and accurate detection of resistance to firstline anti-tubercular drugs in 120 sputum positive AFB smears of pulmonary tuberculosis (PTB) patients.

\section{RESULTS}

The performance of Nitrate Reductase Assay susceptibility testing was rapid and the median time of obtaining results was 10 days.

\section{CONCLUSION}

Direct NRA is simple, easy to perform, rapid, relatively less expensive, without requirement of expensive reagents and sophisticated equipment useful tool suitable for early determination of first-line anti-tubercular drugs namely rifampicin, isoniazid, ethambutol and streptomycin with excellent concordance for INH and RIF resistance and relatively low accuracy for streptomycin and relatively low accuracy for streptomycin with good sensitivity and specificity.

\section{KEYWORDS}

Tuberculosis, Multidrug Resistant, Nitrate Reductase Assay.

HOW TO CITE THIS ARTICLE: Jain N, Jain V, Jain N, et al. Evaluation of mycobacterium tuberculosis drug susceptibility testing by direct nitrate reductase assay. J. Evolution Med. Dent. Sci. 2016;5(77):5759-5761, DOI: 10.14260/jemds/2016/1298

\begin{abstract}
INTRODUCTION
India is the highest tuberculosis burden country in the world and accounts for nearly one fifth $(21 \%)$ of global burden of tuberculosis (TB), which has been a major cause of suffering and death. Early diagnosis of tuberculosis and initiating optimal treatment with chemotherapy via multidrug therapy is recommended. 1 Drug resistance, multidrug resistance (MDR) and extensive drug resistance (XDR) in tuberculosis is a matter of great concern for TB control programs since there is no cure for some multidrug-resistant TB strains of $M$. tuberculosis. ${ }^{2}$ There is concern that these strains could spread around the world, stressing the need for additional control measures, such as new diagnostic methods, better drugs for treatment, and a more effective vaccine. Hence, there is need for diagnosis of the resistant strains early. We evaluated the usefulness of Nitrate Reductase Assay (NRA) as a tool for rapid
\end{abstract}

Financial or Other, Competing Interest: None.

Submission 03-07-2016, Peer Review 11-08-2016,

Acceptance 19-08-2016, Published 26-09-2016.

Corresponding Author:

Dr. Neelu Jain,

Department of Microbiology,

Shyam Shah Medical College,

Rewa-486001, M. P.

E-mail: jainneelu12@gmail.com

DOI: $10.14260 /$ jemds $/ 2016 / 1298$

(c) (i) and accurate detection of resistance to first-line antitubercular drugs.

\section{MATERIAL AND METHODS}

The prospective study was conducted at our institute at Department of Microbiology, Ruxmaniben Deepchand Gardi Medical College, Ujjain after the ethical clearance. All sputum positive (3+) according to RNTCP $^{3}$ (Revised National Tuberculosis Control Programme) grading of AFB smears of pulmonary tuberculosis (PTB) patients were included in the study. Patients with HIV-TB, co-infection and other immunecompromised patients as evident from history, clinical examination and investigations were excluded from the study. After taking written consent and noting of demographic details, the sputum samples of the patient were subjected to microscopy by ZN (Ziehl-Neelsen) stain and graded according to the RNTCP guidelines. ${ }^{3}$ Those samples showing 3+ positivity for AFB on smear were taken and subjected to digestion and decontamination by using Modified Petroff's method. ${ }^{4}$ The sediment obtained after Modified Petroff's method was re-suspended in $3 \mathrm{~mL}$ of sterile distilled water. Out of $3 \mathrm{~mL}$ suspension, $1.5 \mathrm{~mL}$ was used for direct NRA. Direct Nitrate Reductase Assay (NRA) was performed as described by Musa et al. 5 Of the $1.5 \mathrm{~mL}$ suspension, $0.2 \mathrm{~mL}$ was inoculated into four tubes of Lowenstein-Jensen (LJ) medium each, containing potassium nitrate $(1000 \mu \mathrm{g} / \mathrm{mL}) \&$ antitubercular drugs of specific concentration. The critical concentrations 
were $0.2 \mu \mathrm{g} / \mathrm{mL}, 40 \mu \mathrm{g} / \mathrm{mL}, 4.0 \mu \mathrm{g} / \mathrm{mL}$ and $2.0 \mu \mathrm{g} / \mathrm{mL}$ for INH (Isoniazid), RIF (Rifampicin), STR (Streptomycin) and EMB (Ethambutol) respectively. Remaining $0.7 \mathrm{~mL}$ suspension was diluted 1:10 with sterile distilled water, from which $0.2 \mathrm{~mL}$ was used to inoculate 3 tubes of drug free $\mathrm{LJ}$ media with $\mathrm{KNO}_{3}$ to serve as growth control. All inoculated tubes were incubated at $37^{\circ} \mathrm{C}$. The tubes were observed daily for up to 7 days to rule out bacterial contamination and rapidly growing mycobacteria.

On the tenth day, $0.5 \mathrm{~mL}$ of Griess reagent was added to one drug free control tube to observe any colour change (Strong or weak pink). If colour change occurred in the control tube, Griess reagent was added to drug containing tubes and susceptibility results were read. If there was no colour change in the control tube, the remaining control and drug containing tubes were kept back for further incubation. The same procedure was repeated at day 14 and if needed at day 18 using the last i.e. the third growth control tube.

An isolate was considered resistant to a particular antitubercular drug if there was a colour change in the drug containing tube in question, greater than that in the 1:10 diluted growth control tube observed on the same day. If there was no colour change, then the growth was considered susceptible.

\section{RESULTS}

A total of 127 smear-positive (3+) sputum samples were included in the study. Out of the 127 smear-positive (3+) sputum samples, 6 samples showed contaminated growth and 1 sample was niacin negative, thus 7 samples were excluded from data analysis and the 120 samples constituted the sample size. The mean age of patients was 32 years (Range 11 to 70 years). Out of 120 patients, 87 were male and 33 female.

All the results were obtained with in eighteen days duration. The results were obtained on $10^{\text {th }}, 14^{\text {th }}$ and $18^{\text {th }}$ day in 34 (28.3\%), 69 (85\%) and 17 (100\%) patients respectively. The mean duration of detection was 13.4 days. Out of 120 samples, RIF showed 15 resistant and 105 susceptible, INH showed 12 resistant and 108 susceptible, EMB showed 13 resistant and 107 susceptible and STR showed 26 resistant and 94 susceptible.

\section{DISCUSSION}

Tuberculosis continues to be a major cause of morbidity and mortality throughout the world. The most worrisome trend during recent years is an increase in multidrug-resistant (i.e. resistant to RIF and INH) TB strains. Rapid detection of MDR strains is very important to restrict their spread in the population. Current methods for drug sensitivity testing (DST) of MTB are either costly or very slow. So, a cost-effective and rapid drug susceptibility method is required to guide the treatment of TB.1,2,6,7 We evaluated the results of direct NRA Method for first-line anti-tubercular drugs, i.e. RIF, INH, STR, and EMB in 120 sputum samples (3+).
Musa et $\mathrm{l}^{5}$, Mishra, ${ }^{8}$, Sethi et ${ }^{9}{ }^{9}$, Gupta et al $^{10}$ and Anamika et al ${ }^{11}$ all concluded that the performance of Nitrate Reductase Assay susceptibility testing for four first-line anti-tubercular drugs was rapid and the median time of obtaining results was 10 days. These results are quite similar to our study where we found that the mean duration of detection was 13.4 days by NRA method.

RIF and INH are the most important anti-tubercular drugs. Resistance to RIF is almost always associated with multidrug resistance and thus can serve as a marker of MDR-TB if resources are limited. ${ }^{12}$ Hence, it is important to know the resistance to RIF. In our study, RIF showed 15 resistant and 105 susceptible, INH showed 12 resistant and 108 susceptible, EMB showed 13 resistant and 107 susceptible and STR showed 26 resistant and 94 susceptible. This higher resistant show for STR may be due to because STR is a difficult drug to test even by standard methods. This was also seen with other various studies comparing NRA with proportion method (Table 1).

Newer methods like real time PCR for DST are rapid but costly, require equipment and reagents, need skilled technical personnel and are not available everywhere and are restricted to reference laboratories only. Conventional methods like proportion method are cumbersomeness and have longturnaround time.

NRA can be performed in the classical LJ medium, routinely used in TB laboratories, with the addition of $\mathrm{KNO}_{3}$ without need of any sophisticated equipment or expensive reagent, making it a widely-used method. Results are easy to observe by a colour change in the medium. Some strains $(<1 \%)$ of MTB lack nitrate reductase and would create false results since the control would be negative and the test would therefore be invalid. ${ }^{11}$ However, no such strains were encountered in our study.

Secondly, strains of Mycobacterium bovis do not reduce nitrate, for which reason the NRA technique is not applicable. ${ }^{12}$ Further, the ability to reduce nitrate is typical for $M$. tuberculosis, but some other mycobacterial species, like $\mathrm{M}$. kansasii, M. smegmatis, also possess this enzyme, but these are not frequently encountered in humans and they can be easily identified by morphological and biochemical tests. ${ }^{9}$ A very uncommon limitation is that nitrite might be further reduced to nitric oxide, which cannot be detected by the Griess reagents.

To conclude, direct NRA is simple, easy to perform, rapid, relatively less expensive, without requirement of expensive reagents and sophisticated equipments, a useful tool suitable for early determination of first-line anti-tubercular drugs namely rifampicin, isoniazid, ethambutol and streptomycin with excellent concordance for INH and RIF resistance and relatively low accuracy for streptomycin. Further, we recommend, extending the scope of utilising direct NRA for susceptibility testing of second line drugs for M. tuberculosis. 


\begin{tabular}{|c|c|c|c|c|c|c|c|c|c|c|c|}
\hline \multirow[b]{2}{*}{ Studies } & \multirow[b]{2}{*}{ z } & \multicolumn{2}{|c|}{ RIF } & \multicolumn{2}{|c|}{ INH } & \multicolumn{2}{|c|}{ EMB } & \multicolumn{2}{|c|}{ STR } & \multicolumn{2}{|c|}{ Total } \\
\hline & & 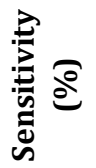 & 胥 & 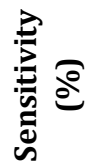 & 莺 & 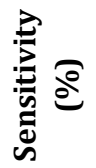 & 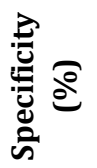 & 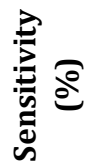 & 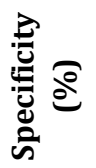 & 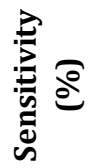 & 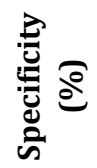 \\
\hline Musa et al ${ }^{5}$ & 121 & 100 & 100 & 93 & 100 & 55 & 99 & 76 & 100 & 82 & 100 \\
\hline Mishra et al8 & 32 & 97 & 97 & 87.5 & 87.5 & NR & NR & NR & NR & NR & NR \\
\hline Sethi et al $^{9}$ & 100 & 100 & 100 & 99 & 100 & 99 & 100 & 100 & 100 & 99 & 100 \\
\hline Gupta et al 10 & 100 & 100 & 100 & 98 & 97.6 & 96 & 83.67 & 74.5 & 98 & 92.59 & 94.56 \\
\hline Anamika et al 11 & 286 & 98.4 & 99 & 97 & 98 & 94.2 & 96 & 88.5 & 87 & NR & NR \\
\hline Coban et al ${ }^{13}$ & 80 & 100 & 100 & 100 & 100 & 92.2 & 100 & 82.1 & 92.3 & NR & NR \\
\hline Visalakshi P et al ${ }^{14}$ & 118 & 94 & 98 & 100 & 98 & NR & NR & NR & NR & NR & NR \\
\hline Agatha Ani et al15 & 70 & 90 & 96.6 & 100 & 100 & 75 & 100 & 66.6 & 98.2 & NR & NR \\
\hline Lemus et al 16 & 320 & 93.9 & 99.1 & 91.7 & 99.1 & 88.0 & 99.1 & 96.5 & 99.1 & NR & NR \\
\hline Angeby et al 17 & 57 & 100 & 100 & 97 & 96 & 75 & 98 & 95 & 83 & 95 & 94 \\
\hline Syre et al 18 & 89 & 94 & 100 & 100 & 95 & 64 & 86 & 88 & 90 & NR & NR \\
\hline
\end{tabular}

Table 1: Comparison of Results of Direct Nitrate Reductase Assay for Drug Susceptibility Testing. (Not reported (NR))

\section{REFERENCES}

1. World Health Organisation. Multidrug and extensively drug-resistant TB (M/XDR-TB): 2010 global report on surveillance and response. 2010. http://whqlibdoc.who.int/publications/2010/97892415 99191_eng.pdf.

2. Affolabi D, Odoun M, Martín A, et al. Evaluation of direct detection of mycobacterium tuberculosis rifampin resistance by a nitrate reductase assay applied to sputum samples in Cotonou, Benin. J Clin Microbiol 2007;45(7):2123-5.

3. Revised national tuberculosis control program. Manual for smear microscopy for detection of acid-fast bacilli. Central TB division, New Delhi 2009.

4. Tripathi K, Tripathi PC, Nema S, et al. Modified Petroff's method: an excellent simplified decontamination technique in comparison with Petroff's method. International J Recent Trends Sci and Tech 2014;10(3):461-4.

5. Musa HR, Ambroggi M, Souto A, et al. Drug susceptibility testing of mycobacterium tuberculosis by a nitrate reductase assay applied directly on microscopy-positive sputum samples. J Clin Microbiol 2005;43(7):3159-61.

6. Canetti G, Fox W, Khomenko A, et al. Advances in techniques of testing mycobacterial drug sensitivity and the use of sensitivity tests in tuberculosis control programmes. Bull World Health Organ 1969;41(1):21-43.

7. Affolabi D, Odoun M, Sanoussi N, et al. Rapid and inexpensive detection of multidrug resistant tuberculosis with the nitrate reductase assay using liquid medium and applied directly to sputum samples. J Clin Microbiol 2008;46(10):3243-5.

8. Mishra B, Muralidharan S, Srinivasa H. Direct drug susceptibility testing of mycobacterium tuberculosis to primary anti-tubercular drugs by nitrate reductase assay. Ind J Pathol Microbiol 2009;52(3):343-4.

9. Sethi S, Sharma S, Sharma SK, et al. Drug susceptibility of mycobacterium tuberculosis to primary antitubercular drugs by nitrate reductase assay. Ind J Med Res 2004;120(5):468-71.
10. Gupta M, Shamma, Singh NP, et al. Evaluation of nitrate reductase assay for direct detection of drug resistance in mycobacterium tuberculosis: rapid and inexpensive method for low-resource settings. Ind $\mathrm{J}$ of Medical Microbiology 2010;28(4):363-5.

11. Gupta A, Sen MR, Mohapatra TM, et al. Evaluation of the performance of nitrate reductase assay for rapid drugsusceptibility testing of mycobacterium tuberculosis in north India. J Health Popul Nutr 2011;29(1):20-5.

12. Martin A, Panaiotov S, Portaels F, et al. The nitrate reductase assay for the rapid detection of isoniazid and rifampicin resistance in mycobacterium tuberculosis: a systematic review and meta-analysis. J Antimicrob Chemother 2008;62(1):56-64.

13. Coban AY, Birinci A, Ekinci B, et al. Drug susceptibility testing of mycobacterium tuberculosis with nitrate reductase assay. Int J Antimicrob Agents 2004;24(3):3046.

14. Visalakshi P, Arora J, Behera D. Evaluation of nitrate reductase assay for rapid detection of resistance to second-line drugs in mycobacterium tuberculosis in a tertiary care hospital. Diagnostic Microbiology \& Infectious Disease 2010;68(3):236-40

15. Ani AE, Dalyop YB, Agbaji O, et al. Drug susceptibility test of mycobacterium tuberculosis by nitrate reductase assay. J Infect Dev Ctries 2009;3(1):16-9.

16. Lemus D, Montoro E, Echemendia $M$, et al. Nitrate reductase assay for detection of drug resistance in mycobacterium tuberculosis: simple and inexpensive method for low-resource laboratories. J Med Microbiol 2006;55(Pt 7):861-3.

17. Ängeby KA, Klintz L, Hoffner SE. Rapid and inexpensive drug susceptibility testing of mycobacterium tuberculosis with a nitrate reductase assay. J Clin Microbiol 2002;40(2):553-5.

18. Syre H, Phyu S, Sandven P, et al. Rapid colorimetric method for testing susceptibility of mycobacterium tuberculosis to isoniazid \& rifampicin in liquid cultures. J Clin Microbiol 2003;41(11):5173-7. 